\title{
Transanal Treatment of Strictured Rectal Anastomosis with a Circular Stapler Device: Simple and Safe
}

\author{
M. Pabst U.Giger M.Senn J.M. Gauer B. Boldog W. Schweizer \\ Department of General Surgery, Kantonsspital Schaffhausen, Schaffhausen, Switzerland
}

\section{Key Words}

Rectal anastomotic stricture $\cdot$ Transanal circular stapling

\begin{abstract}
Background: Several methods of treatment for benign anastomotic strictures after low anterior resection have been described. We report and illustrate a simple, safe, and effective method for treating benign rectal anastomotic strictures by means of a transanal circular stapling device. Methods: Three patients with a clinically significant rectal stricture underwent transanal resection of the fibrous stenosis by a circular stapler device (CEEA stapler 29 or $31 \mathrm{~mm}$ calibre; Tyco Co., USA). Results: No complications occurred. Patients were discharged from the hospital on the first postoperative day. After a follow-up period of 8,12 and 14 months respectively, no recurrence of the stricture was observed. The stool habits of all 3 patients were normal with 1-3 formed, asymptomatic fecal passages per day. Conclusion: Transanal reanastomosis by means of a circular stapler device is a simple and effective method. However, a larger number of patients need to be treated with this relatively new method to draw further conclusions.

Copyright $\odot 2007$ S. Karger AG, Basel
\end{abstract}

\section{Introduction}

Circular stapling anastomosis of the rectum is a safe and quick technique which contributes nowadays to preservation of the sphincter in most rectal cancer operations. However, with the increased application of this technique, colorectal strictures have become a significant problem with the highest reported incidence up to $67 \%$ at the lower rectum [1]. Typically some time after surgery, patients complain of frequent defecation, feeling of residual stool and lower abdominal fullness. Proposed risk factors that may contribute to the development of strictures are ischemia of the anastomosis, incomplete leakage and infection $[2,3]$. Although controversial, many reports have found no difference between hand-sewn and mechanical anastomosis [4]. An ongoing debate exists about the treatment modality of choice for colorectal strictures. Among the different minimally invasive therapeutic treatment options such as transanal dilatation (manual or by bougie), microwave coagulation therapy, transanal incision and argon plasma coagulation, transanal balloon dilatation has been recommended as the preferred treatment option [5]. However, transanal balloon dilatation has only a reported success rate of about $60 \%$ in patients having undergone cancer surgery with an overall complication rate of about $17 \%$ [5]. Among very few case reports about new innovative treatment modal-

W. Schweizer, MD, FACS, FRCS

Head, Department of General Surgery

Kantonsspital Schaffhausen

$\mathrm{CH}-8200$ Schaffhausen (Switzerland)

Tel. +41 5263427 15, Fax +41 5263427 11, E-Mail walter.schweizer@kssh.ch 
Fig. 1. Preoperative water-soluble contrast enema depicts a rectal stenosis (a case 1 , b case 2 ).
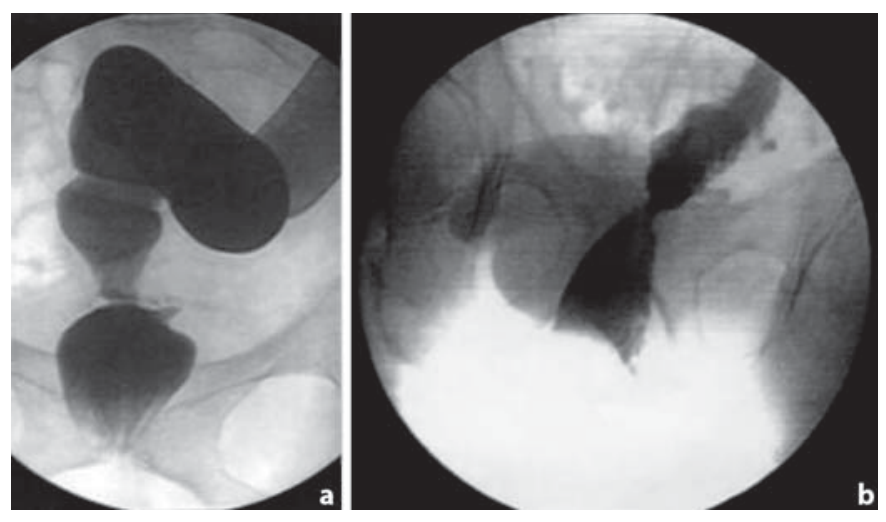

Fig. 2. Schematic illustration: the anvil is introduced with a long clamp (a), the stapler assembled and the head twisted to vertical position (b), then fired (c) and the resected specimen sent for histological evaluation (d).

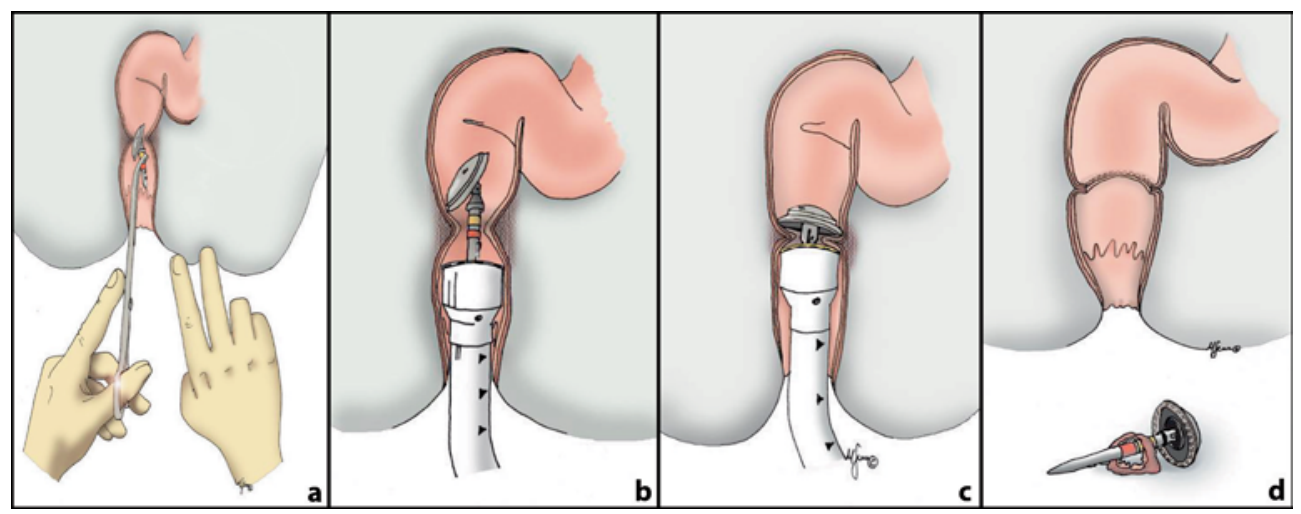

ities with circular staplers for severe colorectal strictures, transanal reanastomosis by means of a circular stapler is reported to be easy and effective [6, 7]. We report our experience with a slightly modified transanal circular stapling technique and describe and illustrate this simple and safe method.

\section{Patients and Method}

Two, 3 and 6 months after the CEEA stapler anastomosis, the 3 patients who had undergone low anterior rectosigmoidal resection for rectal cancer ( 2 patients) and endometriosis ( 1 patient) developed severe symptoms of anastomotic stricture that were proven by endoscopy and radiological contrast examinations. Several attempts of endoscopical dilatation followed by restricturing were unsuccessful so that it was decided to use a transanal stapler resection of the anastomosis.

\section{Methods}

No colonic preparation was performed prior to surgery. All three interventions were undertaken under general anesthesia with the patients placed in the lithotomy position. Cefoxitin $2 \mathrm{~g}$ intravenously was given as a single-shot prophylaxis. After introduction of the anal retractor, the anastomosis was inspected and the stricture quantified. Using a water-soluble contrast medium, before and after surgery, the anastomosis was checked (fig. 1). The CEEA stapler head (29-mm (twice) and 31-mm (once) calibre; Tyco Co., USA) was then introduced. To pass even a severely strictured anastomosis and to reduce the risk of injuring the intestinal wall, the anvil of the stapler device was tilted prior to insertion and then pushed proximal to the stricture with a long clamp whereas the knife- and staple-bearing part being distal to it was introduced in a second step (fig. 2). The anvil was then brought in a vertical position. Introduction, placement and tightening of the stapler were performed under fluoroscopic control (fig. 3). The final result was again controlled by on-table imaging with a water-soluble contrast medium by fluoroscopy (fig. 4). The excised anastomotic stricture was then sent for histological evaluation to exclude a cancerous lesion.

\section{Results}

No complications occurred intra- or postoperatively. All 3 patients were discharged from the hospital on the first postoperative day. Eight, 12 and 14 months after transanal reanastomosis, the patients' stool habits had practically normalized with 1-3 formed, asymptomatic fecal passages per day. During clinical examinations, a soft and homogeneous anastomosis, which could be 
Fig. 3. The stapler is introduced with a long clamp under rectoscopic and fluoroscopic control (a), then the stapler head is put in vertical position under rectoscopic and fluoroscopic control (b), and finally the stapler is assembled with a long clamp to the stapler body and fired under fluoroscopic control (c).

Fig. 4. Postoperative on-table fluoroscopic control with water-soluble contrast medium demonstrates a patent and waterproof reanastomosis (a case $1, \mathbf{b}$ case 2 ).
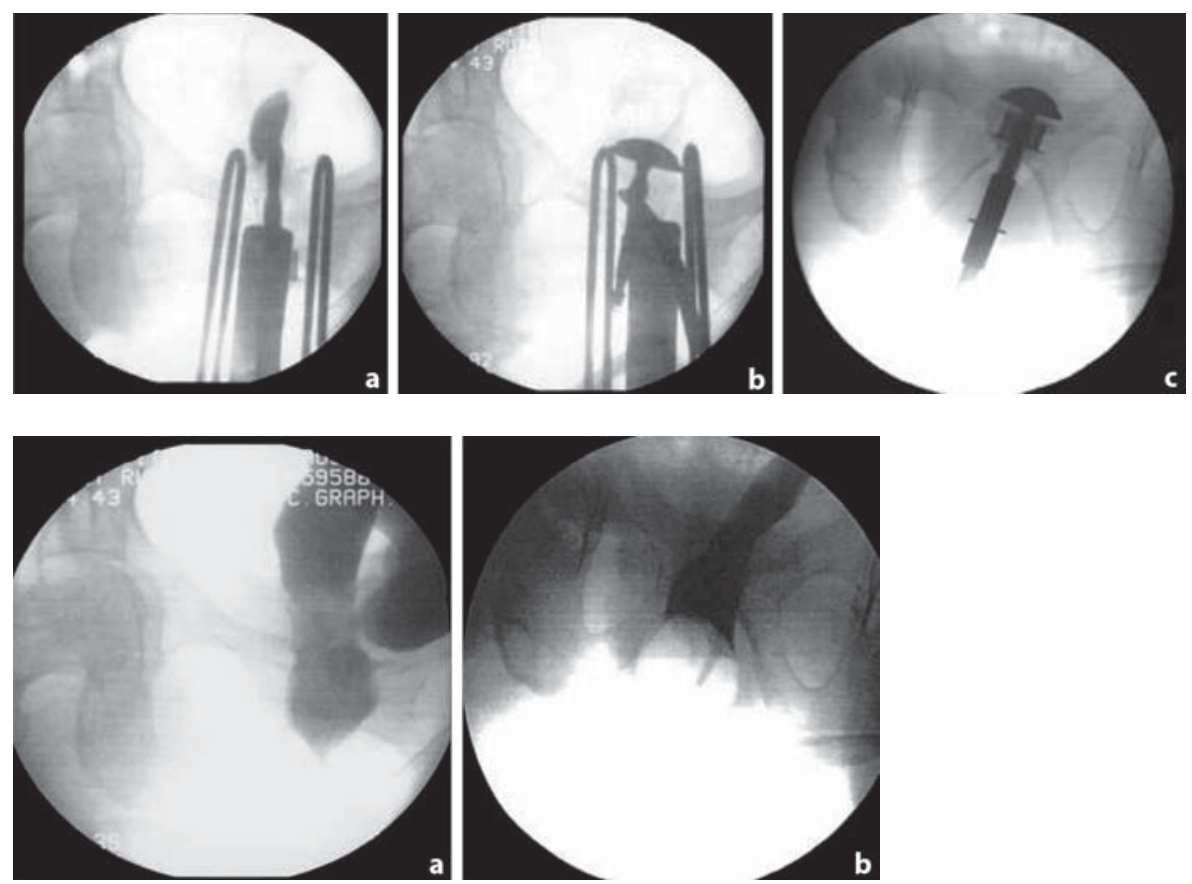

passed with two fingers, was palpated in all 3 cases. Only slight inflammatory changes of the mucosa in the anastomotic region were revealed by colonoscopy.

\section{Discussion}

Abdominal reanastomosis in the lower pelvis is technically challenging with the hazardous possibility of a permanent colostomy. For this reason, transanal minimally invasive procedures are the treatment modalities of choice. Transanal stapler reanastomosis is another simple and safe alternative treatment option. Our slightly modified technique by using an anvil which can be tilted offers the advantage that during stapler insertion, even in severe stenosis, no forced mechanical dilatation is needed. Furthermore, the risk for intestinal injuries during stapler introduction with the risk of perforation is reduced.

This technique is simple and safe. With increasing experience, this treatment modality might be performed as an outpatient procedure. However, more patients need to be treated with these relatively new techniques before final conclusions about safety and long-term follow-up results can be drawn.

\section{References}

1 Luchtefeld MA, Milson JW, Senagore A, Surell JA: Colorectal anastomotic stenosis: results of a survey of the ASCRS membership. Dis Colon Rectum 1989;32:733-736.

2 Smith LE: Anastomosis with EEA stapler after anterior colonic resection. Dis Colon Rectum 1981;141:143-147.
Orsay CP, Bass EM, Firfer B, Ramakrishnan $\mathrm{V}$ : Blood flow in colon anastomotic stricture formation. Dis Colon Rectum 1995;38:202206.

-4 Brain J, Lorber M, Fiddian-Green RG: Rectal membrane: an unusual complication following use of the circular stapling instrument for colorectal anastomosis. Surgery 1981;89: 271-274.

5 Suchan KL, Muldner A, Manegold C: Endoscopic treatment of postoperative colorectal anastomotic strictures. Surg Endosc 2003; 17:1110-1113.
6 Ovnat A, Peiser J, Avinoah E, Charuzi I: A new approach to rectal anastomotic strictures. Dis Colon Rectum 1989;32:351-353.

7 Araki Y, Kishimoto Y, Sato Y, Torigoe S, Kido K: Transanal dilatation using circular stapling for benign rectal stenosis: report of a case. Kurume Med J 2002;49:149-151. 